\title{
A raincoat for Bacillus subtilis
}

\section{Ef}

the protein has

surface-active

properties

allowing the

formation of a water-repellent

coat.

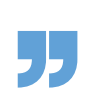

Bacillus subtilis biofilms are waterrepellent and protect bacterial colonies formed on plant roots from environmental threats. BslA, a small secreted protein, is important for biofilm formation and hydrophobicity, but the molecular basis of these functions was unclear. A new study now shows that BslA forms a highly hydrophobic surface layer that shields B. subtilis biofilms.

Immunofluorescence imaging showed that a thin layer of BslA coats the surface of B. subtilis colonies

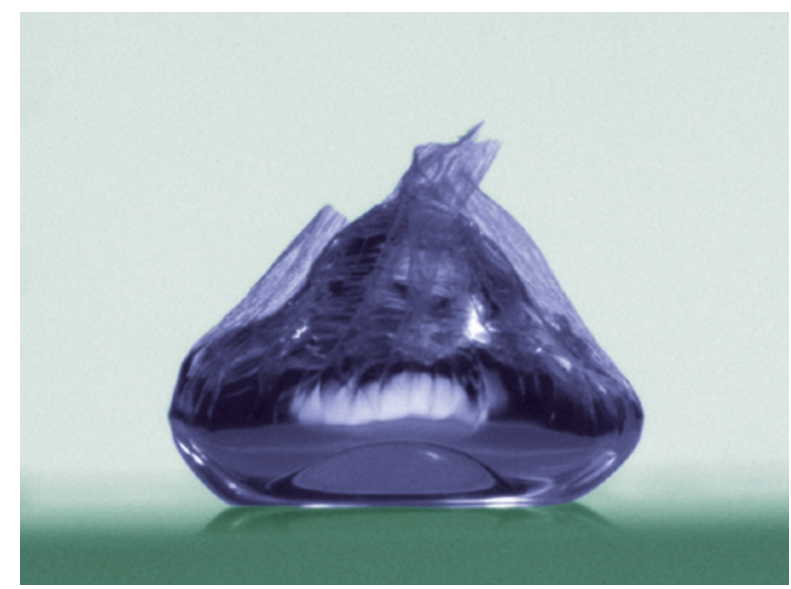

A droplet of a BsIA solution in oil. Image courtesy of N. R. Stanley-Wall, University of Dundee, UK, and false coloured by NPG. grown on agar plates. In biofilms grown at the air-liquid interface of culture medium, BslA forms a thicker layer at the base of the biofilm, and this layer acts like a raft on which the cells sit. Moreover, when water droplets containing purified BslA were expelled into an oil bath by the pendant droplet method, BslA selfassembled into an elastic skin that stably coated the droplet, suggesting that the protein has surface-active properties allowing the formation of a water-repellent coat. Structural analysis of the ordered region of the protein revealed that BslA contains two $\beta$-sheets that form an immunoglobulin-like fold. In addition, a third $\beta$-sheet with numerous exposed hydrophobic amino acids is positioned on top of the fold to form a hydrophobic 'cap'.

Three leucines positioned in the centre of the cap proved to be particularly important for BslA function. Bacteria expressing mutant BslA, in which amino acid 76,77 or 79 was changed from a neutral leucine to a positively charged lysine, no longer formed stable films in the pendant droplet assay. Furthermore, heterologous expression of these mutant
BslA variants did not restore normal biofilm formation in a $b s l A$-knockout strain, as evidenced by the formation of flat, spread-out colonies rather than the wrinkled colonies formed by wild-type B. subtilis. Furthermore, colonies expressing BslA(Leu77Lys) or BslA(Leu79Lys) absorbed water applied to their surface, indicating that these hydrophobic residues are central for BslA function.

Taken together, these results show that BslA acts as a hydrophobin. Hydrophobins are small secreted proteins that contain highly hydrophobic areas and self-assemble into water-repellent coats; such proteins have so far been identified only in fungi. Although BslA differs structurally from fungal hydrophobins, the BslA cap, coated with hydrophobic residues, closely resembles hydrophobic patches found in fungal hydrophobins.

Ursula Hofer

ORIGINAL RESEARCH PAPER Hobley, L. et al.

BslA is a self-assembling bacterial hydrophobin that coats the Bacillus subtilis biofilm. Proc. Natl Acad. Sci. USA 110, 13600-13605 (2013)

FURTHER READING Vlamakis, H. et al. Sticking

together: building a biofilm the Bacillus subtilis way. Nature Rev. Microbiol. 11, 157-168 (2013) 